\title{
The variable-criteria sequential stopping rule: Generality to unequal sample sizes, unequal variances, or to large ANOVAs
}

\author{
Douglas A. FitTs \\ University of Washington, Seattle, Washington
}

\begin{abstract}
The variable-criteria sequential stopping rule (SSR) is a method for conducting planned experiments in stages after the addition of new subjects until the experiment is stopped because the $p$ value is less than or equal to a lower criterion and the null hypothesis has been rejected, the $p$ value is above an upper criterion, or a maximum sample size has been reached. Alpha is controlled at the expected level. The table of stopping criteria has been validated for a $t$ test or ANOVA with four groups. New simulations in this article demonstrate that the SSR can be used with unequal sample sizes or heterogeneous variances in a $t$ test. As with the usual $t$ test, the use of a separate-variance term instead of a pooled-variance term prevents an inflation of alpha with heterogeneous variances. Simulations validate the original table of criteria for up to 20 groups without a drift of alpha. When used with a multigroup ANOVA, a planned contrast can be substituted for the global $F$ as the focus for the stopping rule. The SSR is recommended when significance tests are appropriate and when the null hypothesis can be tested in stages. Because of its efficiency, the SSR should be used instead of the usual approach to the $t$ test or ANOVA when subjects are expensive, rare, or limited by ethical considerations such as pain or distress.
\end{abstract}

The variable-criteria sequential stopping rule (SSR) is a method that allows investigators to conduct nullhypothesis significance tests in stages with a few subjects at a time until the null hypothesis is rejected or the experiment is stopped because the obtained $p$ value is so high that it is unlikely that the experiment will succeed (Fitts, 2010). This method controls the rate of Type I errors near the nominal value, such as .05 , and maintains excellent power. It allows an investigator to progress intuitively through an experiment without investing large amounts of effort, resources, and subjects all at once. The method also stops experiments early when it is obvious that the null hypothesis is not likely to be rejected, and thus, it saves subjects. For this reason, the method is highly recommended for research when a null hypothesis test is appropriate (Frick, 1996) and when it is imperative to use as few subjects as possible because the subjects are rare or expensive or because the treatments cause pain or distress (e.g., experimental surgeries, tests of pain medication). An SSR is not helpful for creating confidence intervals of small size, because small confidence intervals require large sample sizes. The technique is useful mostly when the researcher is satisfied to know whether an effect exists and, if so, in which direction. My original study (Fitts, 2010) did not validate the use of the variable-criteria SSR with more than four groups in an ANOVA or provide guidance on how to use the method if sample size is lost from one or more groups during the experiment. That is the aim of the present study.
The variable-criteria SSR contrasts with the fixed stopping rule, which does not share its benefits. The fixed stopping rule is the customary method for conducting a null hypothesis test, in which the investigator does a power analysis to determine the sample size for the groups, collects data on all subjects, and then analyzes the data with a significance test, such as a $t$ test, once and for all. If the $p$ value is less than alpha (e.g., .05), the investigator rejects the null hypothesis and declares the result significant. If the $p$ value is greater than alpha, little can be concluded. The null hypothesis has not been proven, because the experiment may lack the power to detect an effect of that size. The investigator must not add sample size to the experiment at this point, because the alpha selected for the experiment, such as .05 , is constant only for a single test of the null hypothesis. If the investigator adds sample size and compares the new $p$ value with the same criterion of .05 , the observed rate of Type I errors will rapidly rise above .05 to unacceptable levels. This leads to an increased publication of unreplicable results.

The use of the variable-criteria SSR to control alpha is explained in detail in Fitts (2010). The present method is a variation of previous stopping rules originated by Frick (1998) and modified by Botella, Ximénez, Revuelta, and Suero (2006) and Ximénez and Revuelta (2007). These earlier methods did not control alpha at a constant level and were therefore less powerful than the variable-criteria SSR under certain conditions. To use the variable-criteria SSR, an investigator first conducts a power analysis and

D. A. Fitts, dfitts@u.washington.edu 
determines an experimental model to use in the study using a published graph. The model is a combination of a lower bound; an upper bound; and a number of subjects to add at each step of the experiment, the $n$ added. The lower bound is the number of subjects per group in the first test of the null hypothesis. The upper bound is the number of subjects per group that will not be exceeded in the experiment. Significance is determined by comparing the $p$ value from the first test at the lower bound with two criteria that are determined from a table for the chosen model (Fitts, 2010). If the $p$ value is less than or equal to the lower criterion, the experiment is stopped and the null hypothesis is rejected. If the $p$ value is larger than the upper criterion, the experiment is stopped and the null hypothesis is retained. If the $p$ value is greater than the lower criterion and less than or equal to the upper criterion, the investigator adds $n$ added subjects to each group and conducts another null hypothesis significance test with the augmented sample size. The new $p$ value is then compared with the lower and upper criteria as was just described for the first test. The procedure is repeated until the experiment is stopped because the $p$ value is less than or equal to the lower criterion, the $p$ value is greater than the upper criterion, or the addition of $n$ added subjects would exceed the upper bound of the model. The null hypothesis is rejected only if the first condition is true.

The lower bound in a study should be sufficiently large to convince reviewers that the effect is real, because the investigator will have to stop at that point if the experiment is significant. It should be small enough that subjects are not wasted if the effect size happens to be larger than expected. The upper bound of the model should be large enough that the investigator would generally not wish to invest more resources and subjects anyway. The published tables give a choice of two upper bounds for every lower bound.

The lower criterion will be considerably smaller than the nominal alpha (e.g., it may be .02 instead of .05) in order to maintain the rate of Type I errors at the nominal level (e.g., .05 ) throughout all of the potential tests in the experiment. The value of this lower criterion will be systematically smaller as the number of potential tests to be conducted grows larger (analogous to a Bonferroni test). The upper criterion will be a relatively large probability in the range of .2-.5, and an obtained $p$ above this value indicates that the experiment is unlikely to succeed if further subjects are added, and so the experiment must be stopped. The level of $n$ added is determined solely by the circumstances of the experiment. For example, the investigator may want to ensure optimum efficiency by testing after the addition of each subject per group, or there may be a fixed number of pieces of experimental apparatus that determines the number of subjects that will be tested at each stage.

The published tables of criteria for various models were determined using simulations of large numbers of $t$ tests for independent groups (Fitts, 2010). The Type I error rates were identified when the population means were equal in the simulations, and power was identified when the population means were different. The simulations employed various lower and upper criteria until a pair was found for each model that controlled the overall Type I error rate very close to the nominal level of alpha (e.g., .005, .01, .05, or .10). Thus, there is a different pair of lower and upper criteria in the table for each level of desired alpha, each combination of lower and upper bound, and each level of $n$ added. The investigator selects a model of sample sizes using a graph of the observed power and then identifies the criteria to use by looking up that model in the table (Table 2 of Fitts, 2010).

Loss of sample size is very common in areas such as behavioral neuroscience or medicine, so the behavior of the SSR after loss of sample size needs to be studied. All researchers using SSR procedures should strive to follow the rules to the letter, but sometimes that is impossible. When sample size is lost because of mortality or technical failure, we need to ask two questions: Are my results still valid after the loss of sample size, and what happens if I use additional subjects in the next replication to replace the sample size? Researchers should not be discouraged from using this technique if sample size is lost, and subjects should not be put to unnecessary risk simply to assure that the sample size is equal. The original criteria were determined using twotailed independent-groups $t$ tests, but the same criteria were observed to be valid for any $t$ test (independent or dependent samples with one or two tails) and for a four-group completely random ANOVA. The method would have very limited application if it could not be used with larger numbers of groups in an ANOVA or if the tables had to be rederived for each combination of unequal sample sizes. It would also be very helpful to know whether a planned contrast in a multigroup ANOVA could substitute for the global $F$ as the focus of the stopping rule. For these reasons, I investigated whether the criteria from the previously published table can be generalized to conditions under which sample sizes or variances are unequal or when larger numbers of groups or planned comparisons are required.

\section{METHOD}

\section{Simulations}

Fitts (2010) included simulations of 100,000 repetitions for each data point, because it was essential to determine criteria with the greatest practical stability. Because no new criteria were to be determined for the present investigation and because the present simulations were conducted only to determine the behavior of various models with these original criteria, each simulation to estimate the observed power was conducted 10,000 times and each simulation to estimate the observed alpha was conducted 40,000 times $(4 \times 10,000)$. These rates of Type I errors and power were sufficiently stable compared with the previously published values for this purpose.

Custom programs were designed using the $\mathrm{C}$ programming language in a computing environment that has been described in detail (Fitts, 2010). The data were sampled using a pseudorandom number generator based on Ran2() (Press, Teukolsky, Vetterling, \& Flannery, 1992). The function was seeded on the first call using the system clock so that each sequence would be different. Because of the seeding and the large period of the generator, it is improbable that any long sequence of numbers was correlated or repeated in these simulations. Normalized deviates were then transformed linearly using the desired means and standard deviations to create the generated samples. The skewness and kurtosis statistics of the normalized deviates were verified by sampling a subset of $2^{20}$ random numbers from each simulation. The simulations include results from both pooled-variance and separate-variance $t$ tests (Welch [1947] procedure). The separate-variance $t$ test, which is commonly available on computer statistics packages, yields a more favorable result for 
alpha when variances are unequal, and the new simulations here demonstrate that the Welch approach can help to control alpha with the variable-criteria SSR as well.

The combinations of lower/upper bounds (models) were 3/9, 3/15, 4/10, 4/18, 5/12, 5/19, 6/12, 6/18, 7/14, 7/21, 8/24, 8/32, 9/27, 9/36, $10 / 30$, and $10 / 40$. Selected models were used in some simulations.

The ranges of $n$ added per iteration were 1-6 for the models with lower bounds of 3,4 , and $6 ; 1-7$ for models with lower bounds of 5 and $7 ; 1-8$ for models with a lower bound of 8;1-9 for models with a lower bound of 9; and 1-10 for models with a lower bound of 10 .

The standardized effect sizes (Cohen, 1988) included 0 (null), 0.8 , 1.0, 1.2, 1.4, 1.6, 1.8, and 2.0 for $t$ tests (Cohen's $d$ ) and 0 (null), .40, $.45, .50, .55, .60, .65, .70$, and .75 for multigroup ANOVA $(f)$. 10 .

The four levels of experimentwise alpha were $.005, .01, .05$, and

\section{Data Loss or Unequal Sample Sizes and Variances}

The effects of data loss or unequal sample sizes and unequal variances on the rates of Type I errors and power were determined in two simulations using the independent samples $t$ test with two tails. In the first simulation, I used random data loss with a probability of loss of $20 \%$ for any data point in either group in an experiment. In the second simulation, conditions of equal sample size and variance were compared with conditions of $40 \%$ probability of data loss in only one of the two groups, conditions of heterogeneous variances in a 16:1 ratio, or unequal sample sizes and unequal variances simultaneously.

Data loss in the first simulation was simulated by producing a $20 \%$ probability of deletion of any single score in either of the two groups as the data were generated. The usual rules for this SSR were followed, in that equal numbers of subjects were tested in the two groups at the lower bound, a fixed number of subjects was added to each group at each step regardless of losses, and the experiment was stopped whenever the addition of $n$ added subjects would exceed the upper bound in the group with the larger number of subjects. In this simulation, the losses tended to even out between the groups so that the average sample sizes were similar when a decision was made about the null hypothesis, and the principal effect of the losses was to increase the number of tests to reach a decision.

Unequal variances were created by setting one group's population standard deviation four times greater than the other group's standard deviation. In all equal-variance simulations, the standard deviations were set to 1.0 regardless of the sample size. In simulations with heterogeneous variances, I set constraints such that the average pooled standard deviation in the groups with equal sample sizes would be the same as those in the homogeneous-variance simulations in which the standard deviation was 1.0 and such that the ratio of the standard deviations would be $4: 1$. The solution to these simultaneous constraints was to set the two standard deviations at 0.342997 and 1.371989 in all simulations with heterogeneous variances. This equated the effect sizes for the homogeneous and heterogeneous groups when the sample sizes were equal, because the standardized effect size is dependent on the size of its pooled standard deviation.

In the simulations with the separate-variance (Welch, 1947) approach, these same values for the population standard deviations were used when testing with heterogeneous variances. The Welch approach computes degrees of freedom for the $t$ test such that the greater the heterogeneity of variance, the greater the reduction in degrees of freedom compared with the pooled-variance test.

A combination of the procedures of data loss and unequal variances was simulated for a ratio of standard deviations of $4: 1$ and a simultaneous loss of sample size of $\sim 40 \%$ in only one of the two groups. This led to a marked inequality in sample size at the end of each experiment. In half of the simulations, the standard deviations and sample sizes were positively correlated (the larger standard deviation was in the group with the larger sample size), and in the other half, they were negatively correlated (the larger standard deviation was in the group with the smaller sample size). The simulation was conducted once using the pooled-variance approach and once using the separate-variance approach. A combination of unequal sample sizes and variances is known to produce problems with the pooledvariance $t$ test or ANOVA, with positive correlations greatly reducing power and negative correlations greatly inflating alpha (for a review, see Coombs, Algina, \& Oltman, 1996).

I simulated the rate of Type I errors for the variable-criteria SSR with a two-sample $t$ test across a range of unequal variances $(1: 1$ to $4: 1)$ at different sample size models $(4 / 18,6 / 18,8 / 32$, and 10/40). This simulation was conducted once using the pooled-variance estimate and once using the separate-variance estimate.

\section{New ANOVA Simulations}

I conducted new simulations of completely random ANOVA experiments with 6 or 8 groups using the criteria determined from the previous study for all of the same combinations of alphas, effect sizes, lower and upper bound models, and levels of $n$ added. Additional simulations with 20 groups were conducted with selected models to determine whether alpha was affected with a relatively large number of groups. The observed rate of Type I errors was calculated when the null hypothesis was true (effect size 0 ), and the observed power was calculated when the null hypothesis was false. If the observed alphas from these simulations match the nominal alphas in the simulations $(.005, .01, .05$, or .10$)$, the criteria can be assumed to be valid for use with an ANOVA with that number of groups. Power curves can then be published for use with these ANOVA models using the original criteria. Otherwise, new criteria would have to be determined that provide a better match to the nominal alpha, and new tables of criteria would have to be published for these larger numbers of groups. The latter was obviously undesirable.

\section{SSR With a Planned Contrast}

The use of the variable-criteria SSR with a global ANOVA for multiple groups can be helpful when a significant difference among any combination of the groups is of interest to the research. However, it is often the case that a multigroup ANOVA is designed with several control groups that are all expected to behave in predictable ways in addition to the groups that are of particular interest. An example is an experiment that includes an untreated control group (Group U), a positive control group with a large anticipated difference from the untreated control (Group P), a negative control group with little or no anticipated difference from the untreated control (Group N), and an experimentally treated group (Group E) that is expected to behave like the positive control if the treatment works and the null hypothesis is false. It does not help the researcher to determine whether the experimental group is different from the untreated group if the global ANOVA for the four groups is used as the focus of the stopping rule for the variable-criteria SSR, because the experiment will be stopped as soon as the positive and negative control groups are different from one another. These two groups were included in the design because they are known to be different. What we want is to stop the experiment when the experimental group is different from the untreated control group.

A method for single degree of freedom $F$ tests using planned contrasts is widely discussed in statistics textbooks (e.g., Hays, 1994, pp. 440-443) and is also widely available in computer statistics packages. The method is based on a linear weighting of all of the means in a test such that the sum of the weights equals zero. In the hypothetical experiment just described, we have planned for a large difference to exist between Groups $\mathrm{U}$ and $\mathrm{P}$, and we know that a global ANOVA will rapidly become significant with a small sample size on the basis of the difference between those two groups. What we are most interested in is whether Groups $U$ and $E$ differ and the extent to which Mean E resembles Mean P (i.e., a large effect size). In this instance, we can use a single degree of freedom $F$ to test the difference between Means $U$ and $E$ while ignoring the differences among the other groups. We will use this contrast as the focus of the stopping rule. Thus, our null hypothesis for the contrast will be

$$
\mathrm{H}_{0}:(1) \mathrm{U}+(0) \mathrm{N}+(0) \mathrm{P}+(-1) \mathrm{E}=0,
$$


where the letter represents the population mean and the multiplier in parenthesis is the weight given to that mean in the contrast. The sum of squares for the numerator of the planned contrast equals the square of the sum of the weighted cell means divided by the sum of the ratios of the squared weights to the cell frequencies (Hays, 1994, pp. 440-443). Because the numerator has one degree of freedom, the numerator mean square is identical to the sum of squares. The denominator of the $F$ for the planned contrast is the mean square error for the global ANOVA, and the degrees of freedom for the denominator of the $F$ are the degrees of freedom for the error mean square.

Because the resulting $p$ value is based on the distributions of $F$ with one numerator degree of freedom (equivalently, the $t$ distributions) and because we know that these distributions can be used with the variable-criteria SSR in other circumstances, we can predict that the SSR used in a planned contrast with one degree of freedom will behave in an equally lawful fashion with respect to the observed values of alpha and power. In 40,000 simulations under the null hypothesis and 10,000 simulations under each of eight different effect sizes and 6-10 levels of $n$ added, a planned contrast between Group U and Group E was tested according to the weightings provided above. In the null hypothesis condition, Means $\mathrm{U}, \mathrm{N}$, and $\mathrm{E}$ were all 0 in the population, whereas Mean $\mathrm{P}$ was always equivalent to 1.5 standard deviation units. For analysis of power, the value of Mean $\mathrm{E}$ in the population was varied from 0.8 to 1.5 , whereas those of the other groups were held constant.

In order to compare the results with what would happen if the global $F$ were used as the stopping criterion instead of the planned contrast, the entire experiment was replicated exactly, except that the global $F$ value among the four means was used as the focus of the stopping rule instead of the planned contrast between Groups $U$ and E.

\section{RESULTS}

As in the original study (Fitts, 2010), the number of $n$ added per test in experiments with the variable-criteria SSR did not affect the observed alpha and had only small influences on the power of the test. In order to simplify the presentation, the data are averaged over the 6-10 levels of $n$ added in these experiments. The resulting means were thus based on 60,000-100,000 independent simulations.

\section{$20 \%$ Data Loss}

In the $20 \%$ data loss study, the number of subjects that were actually lost and the number of iterations it took for the simulation to stop depended on the power, which in turn depended on the size of the effect, the sample size, and the nominal alpha. If the null hypothesis was true, many simulations were stopped when the upper criterion was reached on the first iteration while the sample sizes were equal. For example, if the upper criterion was .25, then $75 \%$ of simulations were stopped after the first test. If the null hypothesis was false and power was very high, such as .99 , almost all of the simulations were stopped when the null hypothesis was rejected on the first iteration while the sample sizes were equal. Thus, it was in lower power simulations that stayed for several iterations in the uncertain region that most subjects were lost. These were sometimes lost to one group, sometimes to the other group, and these averaged out. The average of the absolute value of the difference between the sample sizes was about 1 subject or less at the end of the simulations. Thus, subjects that were lost in one iteration tended to be replaced in later iterations.

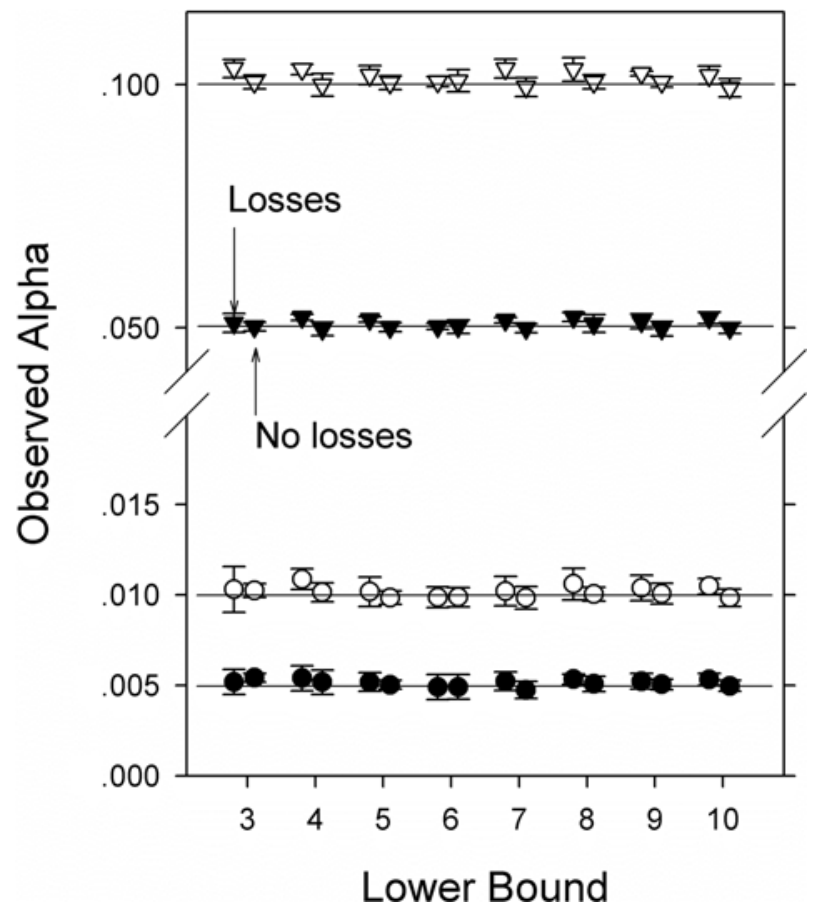

Figure 1. Mean rate of Type $I$ errors observed in simulations of $t$ tests with an effect size of 0 that either had (left of pair) or did not have (right of pair) a $20 \%$ probability of loss of each data point as it was sampled in the variable-criteria SSR. Error bars represent $95 \%$ confidence intervals. Losses tended to balance out through the procedure so that the total sample sizes were approximately equal at the end, and the simulations with losses simply took more iterations than the simulations without losses. This demonstrates that subject replacement during the SSR procedure does not drastically affect the observed alpha.

The rates of Type I errors (observed alpha, mean, and $95 \%$ confidence interval) for the simulations with $\sim 20 \%$ probability of loss of each data point are illustrated for all tested models in Figure 1. Of the 16 available models, only the models with the larger upper bound for each lower bound were included in the study. In Figure 1, the point on the left of each pair is the mean for the simulation with data losses, and the point on the right is the mean for the simulation with no losses. As can be seen, there was excellent agreement between the nominal alpha and the observed alpha in the simulations with no losses (the right-hand point of each pair). At the .05 and .10 levels of nominal alpha, there might be a tiny elevation of observed alpha in the simulations with losses.

The power of the tests when data were lost is illustrated in Figure 2 for selected models (filled symbols), and this was compared with the power of the same models in separate simulations without any loss of subjects (open symbols). Only five models are shown, in order to simplify the presentation. As was explained above, differences in sample size tended to occur when power was lower, and these small differences in sample size caused a small loss of power in all models compared with simulations that suffered no loss of sample size. As power approached 1.0, the probability that the null hypothesis would be rejected on the first test 


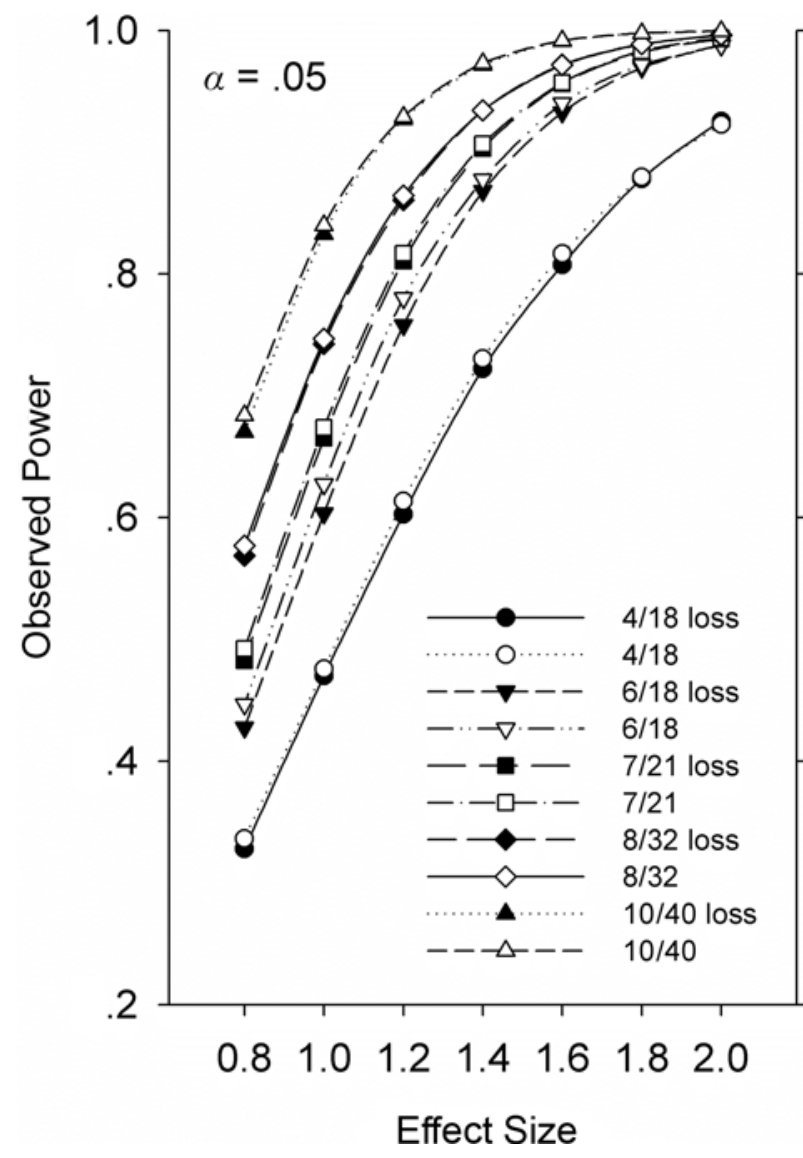

Figure 2. Power observed in simulations of $t$ tests with various effect size that either had (loss) or did not have a $20 \%$ probability of loss of each data point as it was sampled in the variable-criteria SSR. Subject replacement during the SSR does not drastically affect the power of the procedure.

when the sample sizes were equal also approached 1.0, and so the small difference in power disappeared. These power curves without losses fit the previously published power curves for these same models quite well.

The importance of this experiment lies in its implication for data replacement rather than data loss per se, as is explained in the Discussion section.

\section{Unequal Sample Sizes and Unequal Variances}

A second simulation again included a programmed loss of data, except that this time the probability of data loss was $40 \%$ instead of $20 \%$ and the loss was restricted to only one of the two groups of a between-groups $t$ test so that there would be a marked difference between the sample sizes by the time a decision was reached. The simulations included conditions with equal sample sizes and equal variances, conditions with unequal sample sizes and equal variances, and conditions with equal sample sizes and unequal variances. There were two conditions with unequal sample sizes and unequal variances so that the correlation between variances and sample sizes could be either positive or negative. All of these conditions were simulated at all available levels of $n$ added for the models with lower and upper bounds of $4 / 18,6 / 18,8 / 32$, and $10 / 40$. The entire experiment was conducted once using an error term for the $t$ test derived from pooled-variance estimates and a second time using an error term derived from separatevariance estimates (Welch procedure).

The results for the observed alpha for each condition are illustrated in Figure 3 as the mean and a 95\% confidence interval based on the 6-10 simulations with different levels of $n$ added. Data are shown only for the .05 level of alpha, although the results generalize to alphas of $.005, .01$, and .10 as well. The top graph shows the results for simulations using a pooled-variance estimate. In the two groups with homogeneous variances and either equal or unequal sample sizes, the observed alpha matched the nominal alpha quite closely. Thus, a loss of sample size of about $40 \%$ in one of the two groups did not affect alpha when the variances of the groups were equal. Heterogeneous variances with equal sample sizes (labeled Het-Eq in Figure 3) inflated the rate of Type I errors. Compared with that condition, a positive correlation between the variances and sample sizes decreased, and a negative correlation between the variances and sample sizes increased the amount of inflation of alpha. This problem became progressively less pronounced with models of increasing sample sizes. All of this has been reported previously for the $t$ test with the pooled-variance estimate using the fixed stopping rule (Coombs et al., 1996), and the present data demonstrate that the variable-criteria SSR inherits these problems.

This simulation with pooled variances should not be taken to indicate that one can fix the problem of unequal variances and unequal sample sizes simply by increasing the sample size. As an example, I estimated alpha using $100,000 t$ tests using the fixed stopping rule with a total sample size of 80 subjects distributed each of three ways. The standard deviations were 0.342997 and 1.371989 as in Figure 3. With sample sizes of 40 and 40, the observed alpha at the .05 level was .052; with sample sizes of 20 and 60 , the observed alpha was .0022; and with sample sizes of 60 and 20, the observed alpha was .230 . Therefore, the positive correlation between means and variances made it very difficult to find significance (Type II errors), and the negative correlation made it rather difficult to avoid significance (Type I errors) when using the pooled-variance estimate.

By contrast with the pooled-variance approach in the top of the graph, the results using the separate-variance estimates in the bottom part of Figure 3 demonstrate that the problem with heterogeneous variances is greatly improved. As when using the fixed stopping rule, the separate-variance method is obviously to be preferred for the SSR if variances are widely disparate.

The principal conclusions of Figure 3 are that unequal sample sizes do not affect alpha as long as variances are equal, that separate-variance estimates in the standard error term stabilize alpha when variances are heterogeneous, and that a combination of widely heterogeneous variances and unequal sample sizes should be avoided if possible in planned experiments. 

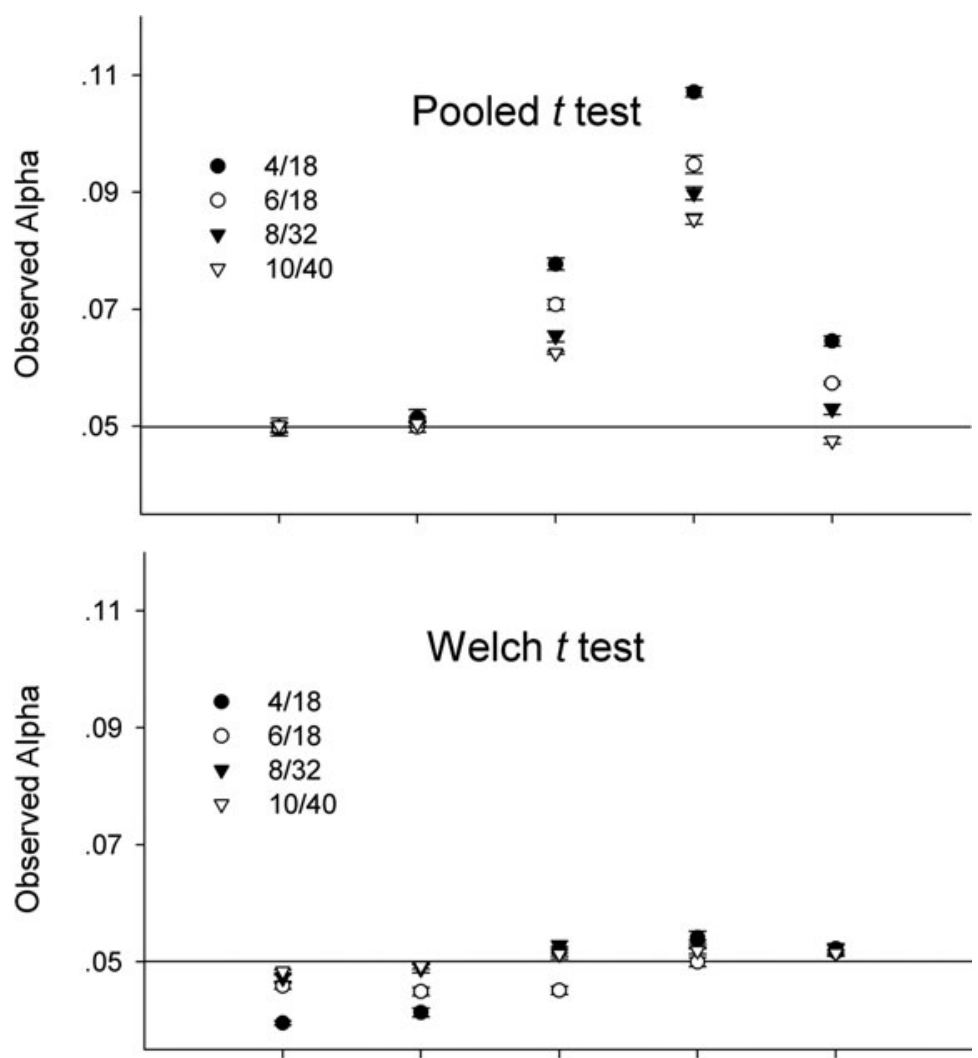

Hom-Eq Hom-Uneq Het-Eq Het-Neg Het-Pos

Variance and Sample Size

Figure 3. Rate of Type I errors during simulations of $t$ tests with the variablecriteria SSR under conditions of equal (Eq) or unequal (Uneq) sample sizes and homogeneous (Hom) or heterogeneous (Het) variances. The top panel shows the pooled-variance method. The bottom panel shows the separate-variance method (Welch test). Sample size loss was $\sim 40 \%$ to one group. The ratio of standard deviations was 4:1 with heterogeneous variances. Neg and Pos, negative or positive correlation between variances and sample sizes.

Figure 4 compares the observed power of these models in each of the simulations with pooled (top) or separate (bottom) error variances under conditions of homogeneous variances and equal sample sizes, homogeneous variances and unequal sample sizes, or heterogeneous variances with equal sample sizes. The standard deviations of the heterogeneous groups $(0.342997$ and 1.371989 , respectively) were contrived so that the effect sizes relative to the pooled standard deviations of both groups were identical to those of the homogeneous groups with standard deviations of 1.0. Thus, they are directly comparable when used with pooled standard deviations. The calculated effect sizes in the other two groups with heterogeneous variances and unequal sample sizes were dependent on the random number of lost subjects at any particular iteration, so they are not directly comparable and are omitted from Figure 4. The filled symbols in the figure represent the power of the variable-criteria SSR under these conditions, and the open symbols in the figure represent the power of the fixed stopping rule as computed from the mean sample sizes at the time of rejection of the null hypothesis for the comparable variable-criteria SSR model. The power of the variable- criteria SSR was at least as great as, and often greater than, the power of the fixed stopping rule for the same average sample size under conditions of heterogeneity of variance or unequal sample size. This was true for both the pooled (top) and the separate (bottom) error terms. This implies that the SSR is more efficient with sample size than is the fixed stopping rule (see the Discussion section).

Despite the apparent similarity of the power of the pooled- and separate-variance methods in Figure 4, there was a small price to pay for the conservation of alpha with the separate-variance approach. The Welch method produces a reduction in the degrees of freedom based on the magnitude of the discrepancy between the variances of the two groups, and this reduces power. What is not obvious in the graphs is that the Welch approach required 1 or 2 additional subjects per group on average before the experiments became significant compared with the pooled-variance approach. Thus, the separatevariance method required a slightly larger sample size to achieve the same power as the pooled-variance method. When using the SSR, this is no problem, because in both cases, animals are added until the experiment is signifi- 

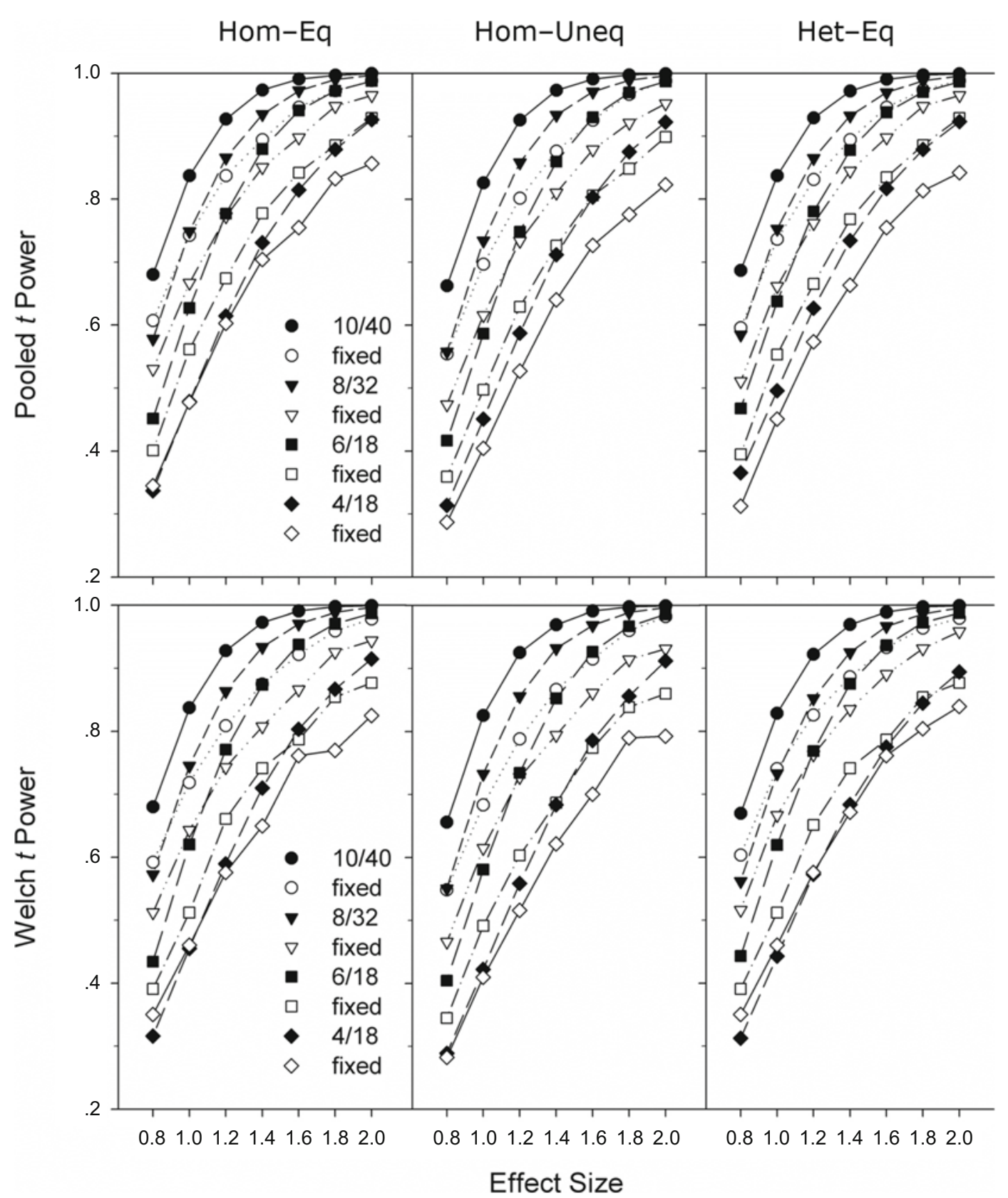

Figure 4. Observed power of the variable-criteria SSR in simulations of $t$ tests under different conditions of equality of variances and sample sizes (filled symbols). Top: pooled-variance method. Bottom: separate-variance method. The power using the fixed stopping rule with the same average number of subjects is shown as the open symbols (fixed). Hom, homogeneous standard deviations, $\sigma_{1}=\sigma_{2}$; Het, heterogeneous standard deviations, $\sigma_{1}=4 \sigma_{2} ; \mathbf{E q}$, equal sample sizes; Uneq, $\sim \mathbf{4 0} \%$ loss of sample size to one of the two groups.

cant. With the fixed stopping rule, additional sample size cannot be added after the first test is conducted, so one must guess the correct sample size before the experiment is conducted. The fixed stopping rule also requires more subjects with the separate-variance approach than with the pooled-variance approach, so the sample-size efficiency of the SSR relative to the fixed stopping rule is preserved with the separate-variance method.

Figure 5 shows the effects of different ratios of standard deviations between 1:1 and 4:1 on the observed alpha of the $t$ test when used with the variable-criteria SSR with a nominal alpha of .05 using either the pooled-variance estimate or the separate-variance estimate (Welch test) approach. The data are presented as the mean of all available levels of $n$ added. The observed alpha rose progressively with greater ratios between the standard deviations with the pooled-variance estimate, although this rise of the observed alpha was less with greater sample sizes. The use of the separate-variance estimate eliminated the problem of increased alpha for the variance ratios tested here. 


\section{Variable-Criteria SSR}

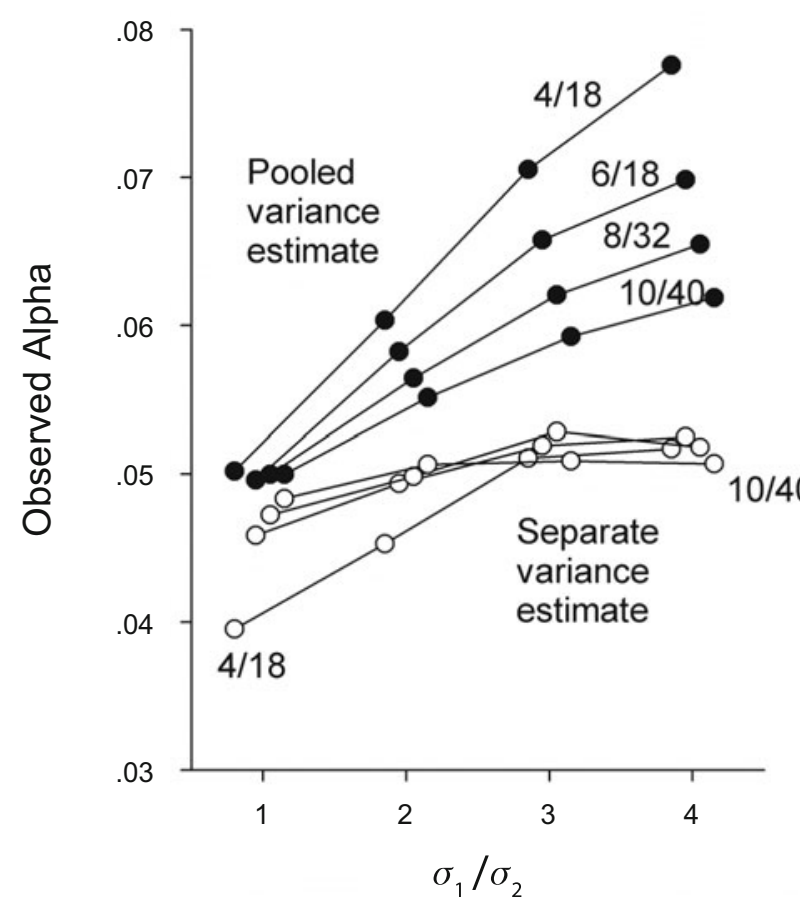

Figure 5. Rate of Type I errors at the .05 level observed in simulations of $\boldsymbol{t}$ tests with the variable-criteria sequential stopping rule (SSR) at different ratios of population standard deviations (1:1, 2:1, 3:1, 4:1). Filled circles: pooled-variance method. Open circles: separate-variance method. Heterogeneity of variances increases alpha in the $t$ test with the pooled-variance method, and the effect is ameliorated by using the separate-variance approach (Welch test).

To add some perspective on the problem, I simulated an experiment that was designed to have an exaggerated inflation of alpha from each of the following: repeated testing with .05 as a lower criterion instead of using a criterion from the table, a small lower bound and a large difference in the variances, a negative correlation between the sample size and variance, and pooled variances instead of separate variances. The effect size was zero, the sample size model was 4/18 with $n$ added from 1 to 6 , the lower criterion was .05 , the upper criterion was .36 , the ratio of standard deviations was $4: 1$, and the group with the larger standard deviation suffered a $40 \%$ loss of sample size. The observed rate of Type I errors was $20.5 \%$ with $n$ added $=1$ and $15.6 \%$ with $n$ added $=6$. These compare with $\sim 10 \%-11 \%$ in the Het-Neg conditions for this sample size model in Figure 3 when the criteria from the table (Table 2 of Fitts, 2010) were used instead of .05 as the lower criterion. As bad as the Type I error rate gets when using the pooled-variance approach with the variablecriteria SSR, it is always much worse if the investigator tests repeatedly with the nominal alpha of .05 instead of using the criteria from the table.

\section{New ANOVA Tests}

The rates of Type I errors, or observed alpha, with different numbers of groups in the simulations with the variable-criteria SSR are displayed in Figure 6. Each point is the mean of 6-10 sets of simulations at different levels of $n$ added with 10,000 repetitions in each set. The error bars give the $95 \%$ confidence interval based on the standard error of the 6-10 points, and these were often smaller than the symbol. The models presented are $4 / 18$, $6 / 18,8 / 32$, and $10 / 40$ (from left to right in each cluster of four points). The numbers of groups were 4, 6, 8, or 20 . The data for 4 groups are reproduced from Fitts (2010). The data for 6 or 8 groups were collected for all 16 available models, and the displayed data for alpha for these few selected models are representative of the entire data set. There appears to be a slight downward drift of alpha with an increasing number of groups in the model using the criteria from Fitts, but these errors are small, and they are all in the more conservative direction when the confidence interval does not include the nominal alpha (i.e., resulting in slightly fewer Type I errors).

Because of the good agreement between the nominal and observed alphas, it is reasonable to use the published criteria from the original study in conducting variablecriteria SSR tests with up to 20 groups. Complete new power curves from the simulations with 6 or 8 groups are displayed in Figure 7, and these can be used as was previously described to select models for study with 6 or 8 groups (Fitts, 2010). Power curves are not presented for the 20-group conditions because the observed power values exceeded $80 \%$ at the .005 level of alpha for all tested effect sizes and sample size models.

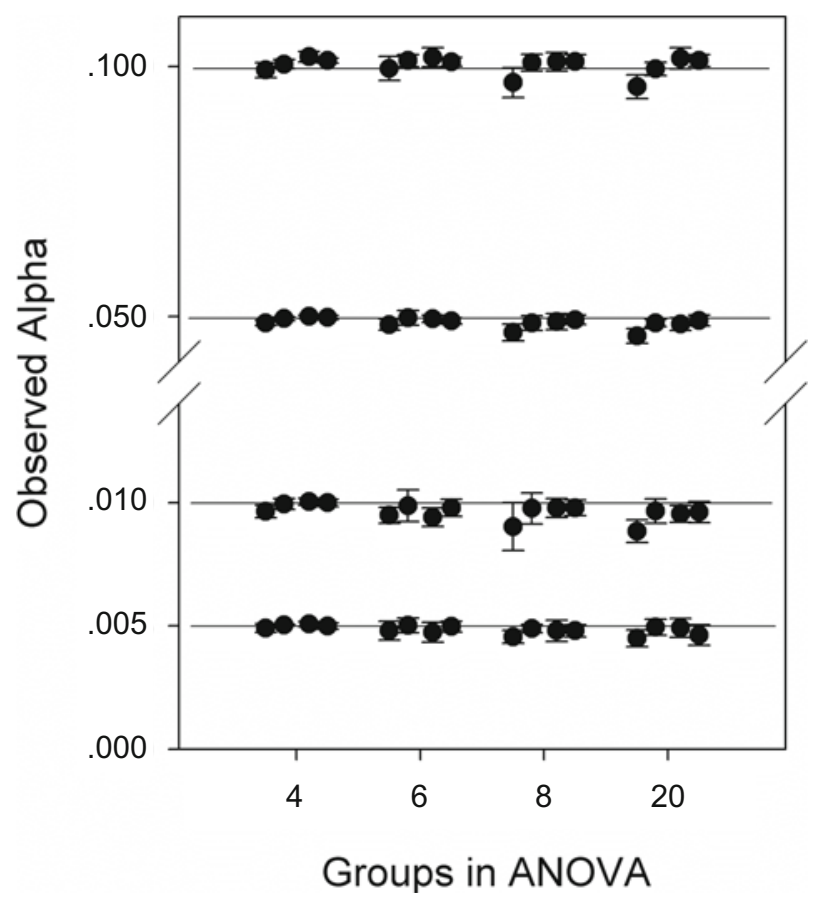

Figure 6. Mean rate of Type I errors observed in simulations using the variable-criteria SSR with 6-20 groups. Error bars represent $95 \%$ confidence intervals. The criteria used were from Table 2 in Fitts (2010). Each cluster of points represents samplesize models (left to right): $4 / 18,6 / 18,8 / 32,10 / 40$. The data for four groups are reproduced from Fitts. 


\section{Six-Group ANOVA}
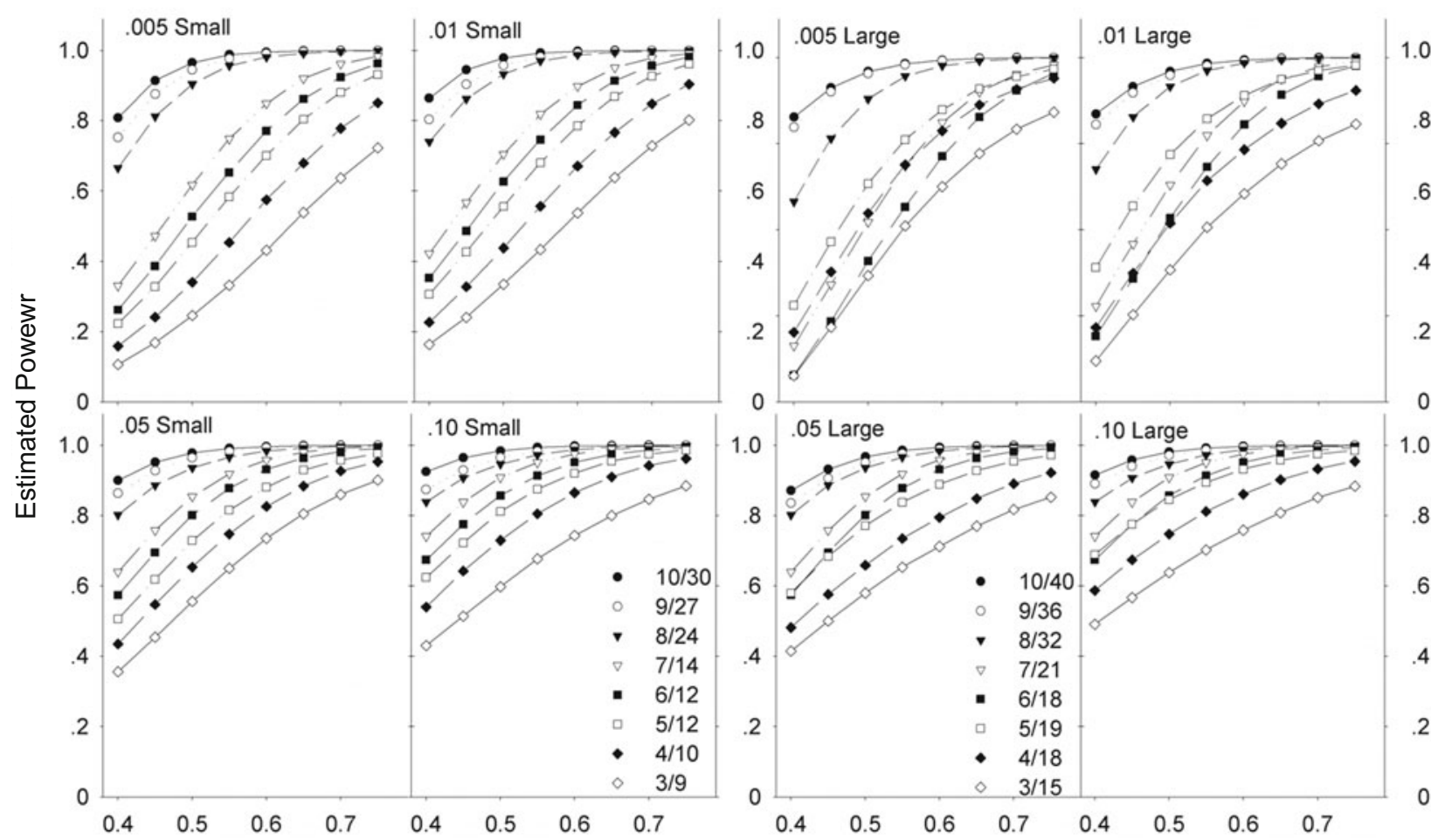

Eight-Group ANOVA
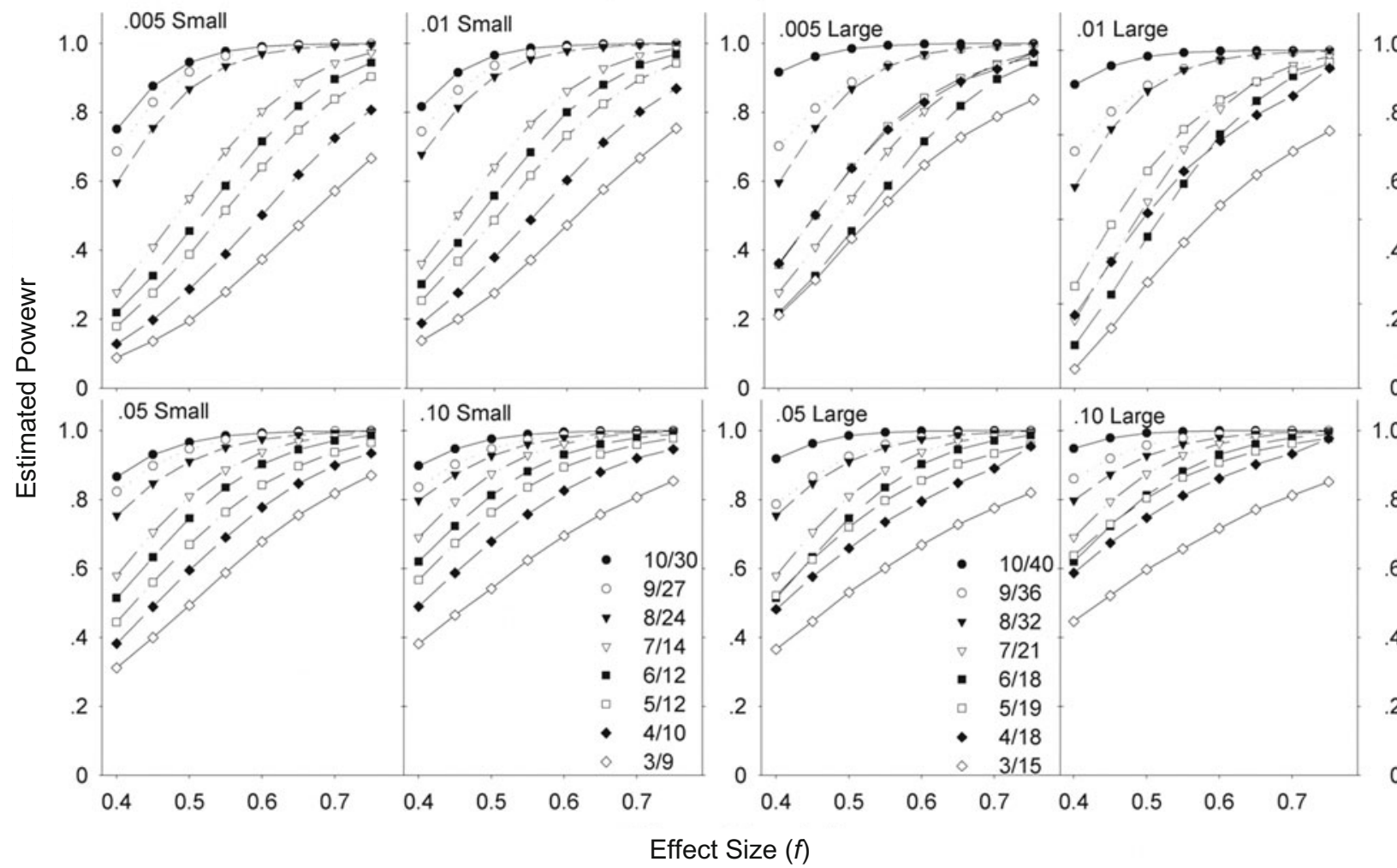

0

Effect Size $(f)$

Figure 7. Power curves for six or eight independent-groups ANOVAs for different levels of experimentwise alpha $(.005, .01, .05, .10)$ at all lower bounds tested in combination with the smaller (left side) and larger (right side) of the two upper bounds for each. The different models (combinations of bounds) are given as lower/upper bound (e.g., 10/30). Given a standardized effect size and a desired alpha and level of power, one can determine the models that will yield at least that level of power. The criteria to use for the selected model at various levels of $\boldsymbol{n}$ added are found in Table 2 of Fitts (2010). 
Table 1

Observed Power of the Two-Group Planned Comparison Simulations and the Power Predicted for Those Simulations From Table 6 in Fitts (2010) at Several Effect Sizes (Cohen's $d$ )

\begin{tabular}{|c|c|c|c|c|c|c|c|c|}
\hline \multirow[b]{3}{*}{ Effect $(d)$} & \multicolumn{8}{|c|}{ Sample Size Model } \\
\hline & \multicolumn{2}{|c|}{$4 / 18$} & \multicolumn{2}{|c|}{$6 / 18$} & \multicolumn{2}{|c|}{$8 / 32$} & \multicolumn{2}{|c|}{$10 / 40$} \\
\hline & Observed & Predicted & Observed & Predicted & Observed & Predicted & Observed & Predicted \\
\hline 0.8 & 0.35 & 0.34 & 0.46 & 0.45 & 0.58 & 0.58 & 0.69 & 0.68 \\
\hline 1.0 & 0.50 & 0.48 & 0.64 & 0.63 & 0.76 & 0.75 & 0.85 & 0.84 \\
\hline 1.2 & 0.64 & 0.61 & 0.79 & 0.78 & 0.87 & 0.87 & 0.93 & 0.93 \\
\hline 1.4 & 0.75 & 0.73 & 0.89 & 0.88 & 0.94 & 0.93 & 0.97 & 0.97 \\
\hline
\end{tabular}

\section{SSR With a Planned Contrast}

The four means in the example for the planned contrast were an untreated group (Group U), a negative control group (Group N), a positive control group (Group P), and an experimental group (Group E). The population values for Groups $\mathrm{U}$ and $\mathrm{N}$ were always 0 in the example, and that for Group $\mathrm{P}$ was always 1.5 standard deviation units. The planned contrast was the difference between Groups $\mathrm{U}$ and $\mathrm{E}$, and this contrast was used as the focus for the variable-criteria stopping rule instead of the global $F$ value among the four means. The population values for Mean E were systematically varied across several simulations, ranging from 0 in the null hypothesis condition to a maximum of 1.5 in the power conditions. When the null hypothesis for the contrast was true, the observed alphas at the $.005, .01, .05$, and .10 levels were very close to the nominal alphas. For example, at the .05 level, the grand mean of four observed alphas and the standard deviations of those four means from simulations of 10,000 independent tests at each of 6-10 levels of $n$ added were $.0479 \pm$ $.0003, .0482 \pm .0009, .0484 \pm .001$, and $.0483 \pm .0009$ for the sample-size models of $4 / 18,6 / 18,8 / 32$, and 10/40, respectively. In this example, using a planned contrast between two means as the focus for the variable-criteria SSR did not elevate alpha. In fact, there may have been a very small bias toward a reduction of alpha.

Figure 10 in Fitts (2010) gives power values for a fourgroup ANOVA for various sample-size models and several effect sizes, and it is used to determine the best sample size model for use with a global ANOVA. That figure will usually underestimate the required sample size for a planned contrast, however. Figure 6 in Fitts for the independent-groups $t$ test is a better predictor of the power of the contrast. For example, the observed power of the planned contrast when the population means for Group E were greater than zero is displayed for several effect sizes in Table 1 along with the power predicted for that contrast from Figure 6 in Fitts for an independent-groups $t$ test. The agreement between the observed and predicted values is sufficient to recommend the use of that previously published table for determining the sample size model to be used to detect a particular twogroup planned contrast in a multigroup ANOVA.

\section{DISCUSSION}

The results of these experiments can be summarized as follows: (1) Losses of sample size up to $40 \%$ in one group in a $t$ test do not affect the observed alpha when the vari- ances are equal. (2) Lost subjects can be compensated for by replacing the subjects in later iterations without a modification of the observed alpha or loss of power. (3) Like the usual $t$ test, the SSR with the pooled error term suffers an inflation of alpha with increasing ratios between the standard deviations. The use of a separate-variance approach eliminates this problem. (4) The variable-criteria SSR as previously published can be used safely without modification in completely random ANOVA models with at least 20 independent groups. (5) The SSR is at least as powerful as the fixed stopping rule and is often more powerful. (6) A single degree of freedom planned contrast can be used as the focus of the SSR instead of the global $F$ to allow the experiment to be stopped when a specific effect of interest becomes significant. Overall, the variable-criteria SSR shares many of the same advantages and drawbacks as the usual ANOVA for real-world experiments while also allowing investigators to ensure that the minimum number of subjects will be used on average. I want to emphasize that in all cases in which the variable-criteria SSR is more powerful than the fixed stopping rule with the same number of subjects (e.g., Figure 4), this implies that the SSR would require fewer subjects than the fixed stopping rule with the same power. Thus, the SSR is often more efficient with subjects and is to be preferred when the use of subjects is constrained by ethics, availability, or expense. The implications of these findings for the replacement of lost subjects, for heterogeneous variances, and for planned contrasts and factorial ANOVAs are discussed below.

\section{Replacement of Lost Subjects}

When subjects were allowed to be lost in either of the two groups, the experiments required more iterations to reach a decision, but the observed alpha was similar to the nominal alpha (Figure 1) and the power of the test was similar to the same model without losses (Figure 2). This is an important finding, because these sequences of sample sizes might have occurred either by there being an equal probability of loss in the two groups when the probability of assignment of subjects to the two groups was equal or by there being an unequal probability of loss in the two groups when there was a larger probability for inclusion of subjects in the group with the larger probability of loss. For example, suppose that a behavioral neuroscience experiment includes animals with a particularly difficult brain surgery in one group and a sham surgery in the other group. It would be more likely that losses to sample size would occur in the group with the difficult 
surgery because the preparations are more likely to fail for one reason or another. The investigator may assign a higher probability for random inclusion of animals in the difficult surgery group in order to compensate for the anticipated losses and to achieve approximately equal sample sizes for successful surgeries and sham surgeries at the end of the experiment. On the basis of Figures 3 and 4, this compensatory procedure should have little effect on the observed rate of Type I errors or on the power of the test.

\section{Heterogeneous Variances}

When used with a pooled estimate of the error variance, unequal variances cause an increase in the rate of Type I errors with the variable-criteria SSR just as they do with the usual ANOVA or $t$ test. This situation is not improved by switching to nonparametric tests such as the MannWhitney-Wilcoxon or the Kruskal-Wallis rank tests (per unpublished data for variable-criteria SSR; Zimmerman 1999, 2000), so investigators are usually better off staying with the parametric test. Investigators who do need nonparametric tests should contact the author for tables of criteria specific to these tests. Some nonlinear transformations may help to stabilize variances, but the scientific or biological meaning of the transformed variables may not always be clear. The separate-variance $t$ tests suggested by Welch (1947) and Satterthwaite (1946) can be useful, and these methods are reviewed in Coombs et al. (1996). The $p$ values from these $t$ tests can easily be used with the variable-criteria SSR, and this eliminates the problem with unequal variances for variance ratios up to 16:1. One can also consider more modern robust statistical methods that are less influenced by violations of these particular assumptions (Erceg-Hurn \& Mirosevich, 2008). Certainly, the problems with Type I error rates or power get much worse when the heterogeneity is compounded by markedly unequal sample sizes. Fortunately, the unequal sample sizes can be avoided with the variable-criteria SSR by adding subjects proportionately in sequential tests to equate the sample sizes as was discussed in the previous section.

Figure 4 displays the power curves for the four samplesize models across a range of effect sizes for a $t$ test with either a pooled- or a separate-variance estimate. The filled symbols represent the power of the variable-criteria SSR, and it is compared with the power of the fixed stopping rule in the open symbols. To illustrate what this means, consider the data for the circles at the top of the lefthand side of either left-hand graph in Figure 4 (homogeneous variances and equal sample sizes, 10/40 model). The filled circle is the observed proportion of the time after all simulations with an effect size of 0.8 that the null hypothesis was rejected at the .05 level with the $10 / 40$ model (power $=.68$ ). For all of the simulations in which the null hypothesis was rejected, the mean total number of subjects used was about 16 or 17 per group in this equal sample size condition (data not shown). A simple power analysis for these same parameters with a sample size of 17 per group and an alpha of .05 yields a power of .62 for the traditional fixed stopping rule, so the fixed stopping rule is less powerful with the same number of subjects.
The fixed stopping rule would require a sample size of 20 per group to exceed the power of the SSR (power $=$ .69 with $n=20$ per group). Thus, the experiment with the variable-criteria SSR has saved 4-6 subjects in the experiment (groups combined) in order to achieve this level of power. This efficiency is not undone by unequal sample sizes, by mildly heterogeneous variances, or by the use of a separate-variance estimate $t$ test (bottom of Figure 4), and it is an excellent reason to use the variable-criteria SSR instead of the fixed stopping rule in studies that involve pain or distress to the subjects.

\section{SSR With a Planned Contrast or Factorial ANOVA}

The results with 6-20 groups in a one-way ANOVA encourage the notion that the same table of criteria may generalize to a wide variety of ANOVA models. I can recommend the technique for any number of groups between 2 and 20 in a one-way analysis, and I see no strong trend to indicate that it would not also control Type I errors with more groups. This has several interesting implications.

The variable-criteria SSR technique can be used with a particular planned contrast instead of with a global ANOVA as its focus. In a one-way ANOVA with $G$ groups, there are $G-1$ degrees of freedom for the total betweensubjects sum of squares and always no more than $G-1$ different independent (orthogonal) contrasts among the means that can be made as planned comparisons (see, e.g., Hays, 1994, pp. 440-443). Each of these contrasts generates an independent $F$ ratio with 1 degree of freedom. Because the SSR works with the $p$ value from this distribution, and the SSR has no way of being influenced by the fact that the $p$ was generated from a planned contrast instead of from a $t$ test, it stands to reason that any of these $F$ s can be used as the focus for the variable-criteria SSR technique instead of the omnibus ANOVA. This allows the experiment to be stopped when the effect of interest represented by the planned contrast is significant.

When positive and negative controls are included in an experiment in order to scale a new treatment with respect to known treatments, it is best to test a planned contrast among the means of interest instead of the global $F$ as the focus of the stopping rule. This will stop the experiment when the means of the experimental group and a control group become significantly different, instead of when the means of the positive and negative controls become significantly different. Alpha will be held stable at approximately the nominal value that is determined before the experiment is conducted, and power will be similar to that of Figure 6 of Fitts (2010). Thus, that previously published figure can be used to design the best sample-size model to use for planned contrasts such as the example presented here.

It is important to understand why the global $F$ among the four groups should not be used as the focus of the variable-criteria SSR when a positive control group of minor importance is included in the multigroup ANOVA (as in our example), and why the curves in Figure 10 of Fitts (2010) for the four-group ANOVA should not be used to plan the sample-size model for such an experiment. The 
null hypothesis condition for the planned contrast between the experimental group (Group E) and the untreated control group (Group U) was not also a null hypothesis condition for the global ANOVA because the population mean value for Group P was always 1.5 standard deviations instead of 0 . That is, we know that the positive control group will almost always have a large effect. In the example, this meant that the overall effect size (Cohen's $f$ ) for the global ANOVA was .65 in the null hypothesis condition for the planned contrast (i.e., when Population Mean E $=0$ ) and was .75 when Group E had its maximum value (Population Mean $\mathrm{E}=1.5$ ). The power curves for the ANOVA in Figure 10 of Fitts predict a rate of rejection of the null hypothesis (i.e., power) of greater than .7 when Mean $\mathrm{E}=0$ and greater than .8 when Mean $\mathrm{E}=1.5$, and this was what I observed in the simulations using the global $F$ value as the focus of the stopping rule (observed rate of rejection of .78 when Mean $\mathrm{E}=0$ and .87 when Mean $\mathrm{E}=1.5$ ). Using the global $F$ value as the focus will often stop the experiment before a weaker but more interesting contrast between Means U and E can become significant. Using the planned contrast instead of the global $F$ as the focus of the stopping rule solves this problem. The power of the planned contrast may be less than the power of the global ANOVA, so the sample-size model for a planned contrast should be selected using Figure 6 for two groups instead of Figure 10 in Fitts for four groups, and the anticipated effect size of the contrast should be calculated using the two-group contrast instead of the global fourgroup effect.

By similar logic, if an experiment has a completely random factorial design structure, the variable-criteria SSR can be focused on any one of these component $F$ values instead of on the total between-subjects $F$. Any completely random factorial design can always be analyzed instead as a one-way ANOVA with planned comparisons, so the above analysis would apply. Furthermore, the separate effects in a factorial structure are completely independent, so the SSR has no way of being influenced by the fact that one factor may or may not be crossed with an independent factor. If an SSR can be used with a one-way ANOVA with four groups, it can also be used with exactly that same four-group factor when it is crossed with a completely independent factor.

For example, if the experiment includes an important treatment factor and a control factor, such as batch of drug or time of day, the stopping rule can be based on the treatment of interest instead of on uninteresting differences on the control factor. Even the interaction term can serve as the focus of the procedure as long as this is decided before the experiment begins and it is the only $p$ value that is considered when decisions are made about adding subjects in the SSR. One should keep in mind that the procedure has not yet been validated with more than 19 degrees of freedom in the numerator term.

It is important that one and only one $p$ value can serve as the focus of the procedure for stopping the experiment. For example, the observed alpha in this procedure would be unknown if an investigator continued to add subjects until the effects for Factor A and Factor B were both significant.

\section{Limitations and Future Research}

The SSR models reported here were intended for use in small-sample experiments in biopsychology or medicine, often with animal subjects, where the anticipated effect size is quite large and it is important to limit the sample size as much as possible for ethical reasons. Other SSR approaches have been presented that are effective with large-sample techniques (Botella et al., 2006; Frick, 1998; Ximénez \& Revuelta, 2007). Future research could extend the variable-criteria method to smaller effect sizes to assure users of a relatively constant observed alpha. New studies should also be conducted to examine the effects of a combination of heterogeneous variances and nonnormal distributions with the SSR. One would hope that the SSR is no worse than the usual ANOVA with these conditions that badly inflate alpha and reduce power, but the behavior of the SSR under these particular conditions has not yet been demonstrated.

\section{AUTHOR NOTE}

Correspondence concerning this article should be addressed to D. A. Fitts, Office of Animal Welfare and IACUC, University of Washington, Box 357160, Seattle, WA 98195 (e-mail: dfitts@u.washington.edu).

\section{REFERENCES}

Botella, J., Ximénez, C., Revuelta, J., \& Suero, M. (2006). Optimization of sample size in controlled experiments: The CLAST rule. Behavior Research Methods, Instruments, \& Computers, 38, 65-76.

CoHen, J. (1988). Statistical power analysis for the behavioral sciences (2nd ed.). Hillsdale, NJ: Erlbaum.

Coombs, W. T., Algina, J., \& Oltman, D. O. (1996). Univariate and multivariate omnibus hypothesis tests selected to control Type I error rates when population variances are not necessarily equal. Review of Education Research, 66, 137-179.

Erceg-Hurn, D. M., Mirosevich, V. M. (2008). Modern robust statistical methods: An easy way to maximize the accuracy and power of your research. American Psychologist, 63, 591-601.

FiTTs, D. A. (2010). Improved stopping rules for the design of efficient small-sample experiments in biomedical and biobehavioral research. Behavior Research Methods, 42, 3-22.

FrICK, R. W. (1996). The appropriate use of null hypothesis testing. Psychological Methods, 1, 379-390.

FRICK, R. W. (1998). A better stopping rule for conventional statistical tests. Behavior Research Methods, Instruments, \& Computers, 30, 690-697.

Hays, W. L. (1994). Statistics (5th ed.). Orlando: Harcourt Brace \& Company.

Press, W. H., Teukolsky, S. A., Vetterling, W. T., \& Flannery, B. P. (1992). Numerical recipes in C: The art of scientific computing (2nd ed.). Cambridge, MA: Cambridge University Press.

Satterthwaite, F. E. (1946). An approximate distribution of estimates of variance components. Biometrics Bulletin, 2, 110-114.

Welch, B. L. (1947). The generalization of Student's problem when several different population variances are involved. Biometrika, 34, 28-35.

Ximénez, C., \& Revuelta, J. (2007). Extending the CLAST sequential rule to one-way ANOVA under group sampling. Behavior Research Methods, Instruments, \& Computers, 39, 86-100.

Zimmerman, D. W. (1999). Type I error probabilities of the WilcoxonMann-Whitney test and Student $t$ test altered by heterogeneous variances and equal sample sizes. Perceptual Motor Skills, 88, 556-558.

Zimmerman, D. W. (2000). Statistical significance levels of nonparametric tests biased by heterogeneous variances of treatment groups. Journal of General Psychology, 127, 354-364.

(Manuscript received February 19, 2010; revision accepted for publication June 18, 2010.) 